\section{Neoplastic lymphangiosis of the upper aerodigestive tract simulating field cancerization: histopathological analysis, surgical limits and literature review}

\author{
Franco Mattavelli ${ }^{1}$, Natalia Pizzi ${ }^{1}$, Elisabetta Pennacchioli ${ }^{2}$, Stefano Radaelli ${ }^{2}$, \\ Pasquale Quattrone ${ }^{3}$, Davide Mattavelli ${ }^{1}$, Andrea Fior ${ }^{4}$, and Silvana Pilotti ${ }^{3}$ \\ 'Otolaryngology Unit, ${ }^{2}$ Surgical Department, and ${ }^{3}$ Pathology Unit, IRCCS Istituto Nazionale \\ dei Tumori, Milan; ${ }^{4}$ Maxillofacial Surgery Clinic, Università degli Studi di Verona, Verona, Italy
}

\section{ABSTRACT}

Neoplastic lymphangiosis is defined as extensive embolic spread of cancer cells in the lymphatic vessels often without any evidence of a mass. Instead, field cancerization is defined by the presence of multifocal neoplastic lesions in a mucosal field previously exposed to mutagenic factors. In this case report, this oncological entity was suggested by the wide extent and multifocality of the disease and by the patient's exposure to risk factors. Instead, the pathological slides revealed the integrity of the mucosa and the presence of widespread embolic metastasis to lymphatic vessels. Thus, the diagnosis was changed to neoplastic lymphangiosis. This clinical presentation is a negative prognostic factor, and surgical treatment is ineffective because of the impossibility to obtain adequate free margins. The present case underlines the poor prognosis of such locally advanced cancer and the importance of recognizing it early so that the treatment approach can be adapted.

\section{Introduction}

The metastatic spread of tumor cells is the most lethal aspect of cancer and often occurs through the lymphatic vessels. Neoplastic lymphangiosis is the wide and homogeneous spread of cancer cells in the lymphatic vasculature of an anatomic district with or without the clinical evidence of a mass ${ }^{1}$.

We have dealt with a peculiar case in which we observed widespread cancerization of the upper aerodigestive tract (UADT) previously irradiated for head and neck cryptogenic squamous cell carcinoma (SCC). Because of the patient's previous exposure to alcohol, smoke and radiation, we made a tentative diagnosis of field cancerization. Instead, histological analysis showed complete integrity of the mucosa with widespread neoplastic embolization of the lymphatic vessels. This morphological evidence led to a diagnosis of neoplastic lymphangiosis.

\section{Case report}

This is the case of a male patient who was a heavy drinker and heavy smoker (20 cigarettes/day for 30 years). At the age of 55 years, clinical evidence of massive right cervical lymphadenopathy (levels $2 \mathrm{a}$ and 3 ) led the patient to undergo a fine-needle aspiration biopsy, which documented SCC metastasis. Pharyngolaryngoscopy with multiple biopsies was negative for malignant cells. After a right radical neck dissection with nodes that proved positive for metastatic cells from SCC, the patient received postoperative cobalt radiotherapy to both laterocervical sites (54 Gy) and to the pharyngeal/laryngotracheal area (27 Gy).

Seven years later, a CT scan showed a mass in the left lung with lymphadenopathies of the homolateral hilum. The preoperative histological diagnosis was primary SCC.
Key words: field cancerization, squamous cell carcinoma, neoplastic lymphangiosis.

Correspondence to: Franco Mattavelli, MD, Via Galbiati 6, 20864 Agrate Brianza (MB), Italy. Tel +39-348-3579926; email franco.mattavelli@yahoo.it

Received September 16, 2011; accepted January 26, 2012 
The patient underwent a left pneumonectomy and postoperative radiotherapy by linear accelerator (50 Gy) to the mediastinum and left hilum with partial overlap in the jugular region $(1-2 \mathrm{~cm})$ of the previously irradiated field.

Another 5 years later, the patient first came to our attention at the National Cancer Institute in Milan because of worsening dysphagia and odynophagia. Pharyngolaryngoscopy revealed diffuse post-actinic fibrosis and an infiltrating solid lesion $(15 \mathrm{~mm})$ of the right glossoepiglottic vallecula. Driven by the preoperative biopsy result, we performed a total laryngectomy. The final diagnosis was undifferentiated carcinoma. All resection margins (hypopharyngeal and esophageal wall, mucosa of tongue, larynx and trachea up to the fourth ring) showed neoplastic spread to the submucosal lymphatic vessels as is clearly visible in Figures 1, 2 and 3. The tumor was staged pT4 Nx Mx G3.

In the postoperative period the patient needed surgical repair of a large pharyngostoma by means of a pectoral myocutaneous pedicled flap. During the procedure several biopsy samples of macroscopically normal mucosa were obtained along all the margins of the surgical, previously irradiated field. The histopathological report confirmed undifferentiated SCC in all samples in the presence of integrity of the mucosa and widespread neoplastic embolization of the lymphatic vessels. The patient was administered palliative chemotherapy consisting of 4 cycles of cisplatinum and 5-FU according to the Al-Sarraf regimen. Local relapse in the form of a single neoplastic mass occurred 6 months later, determining progressive stenosis of the pharyngoesophageal junction which led to the patient's death.

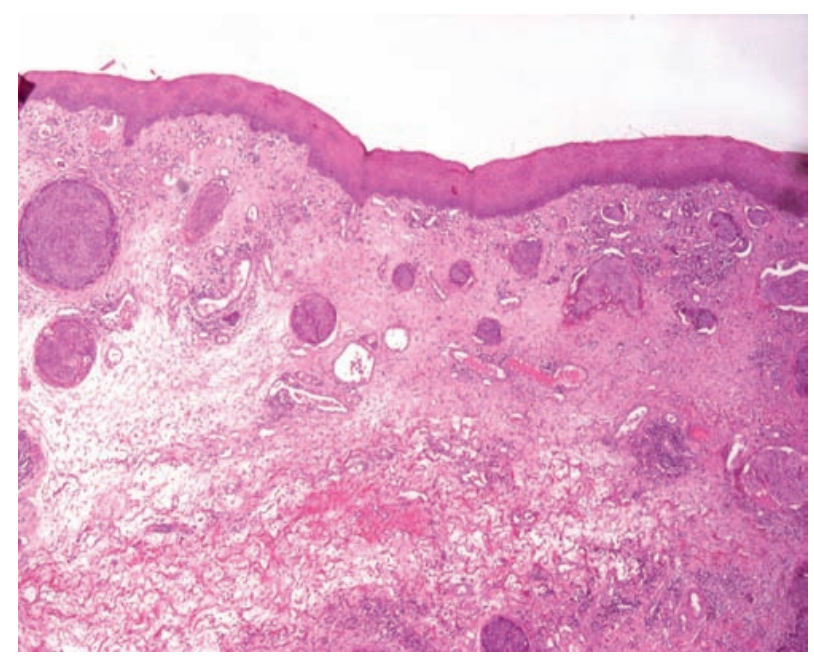

Figure 1 - Pharyngoesophageal mucosa. In this histological section (as in the ones of Figures 2 and 3 ) the mucosal surface appears completely uninjured; by contrast, widespread neoplastic embolization of the submucosal lymphatic vessels is clearly visible.

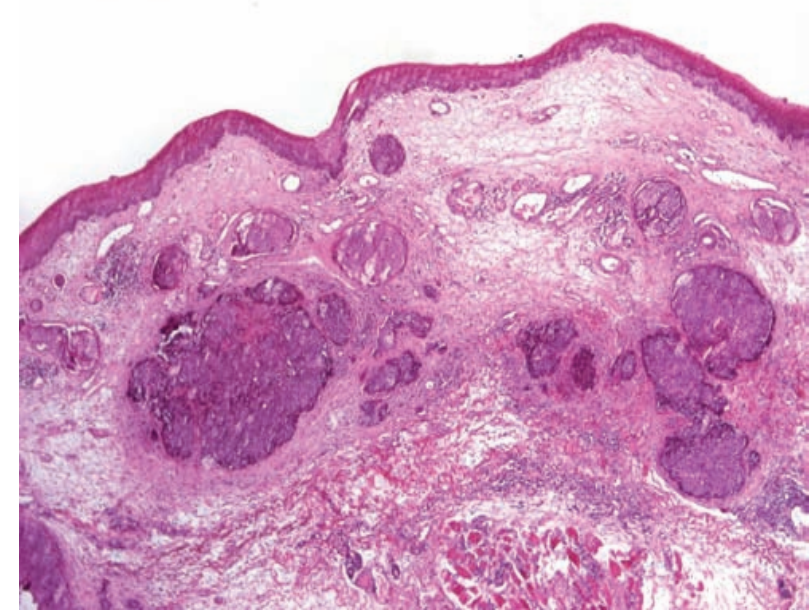

Figure 2 - Margin of the tongue.

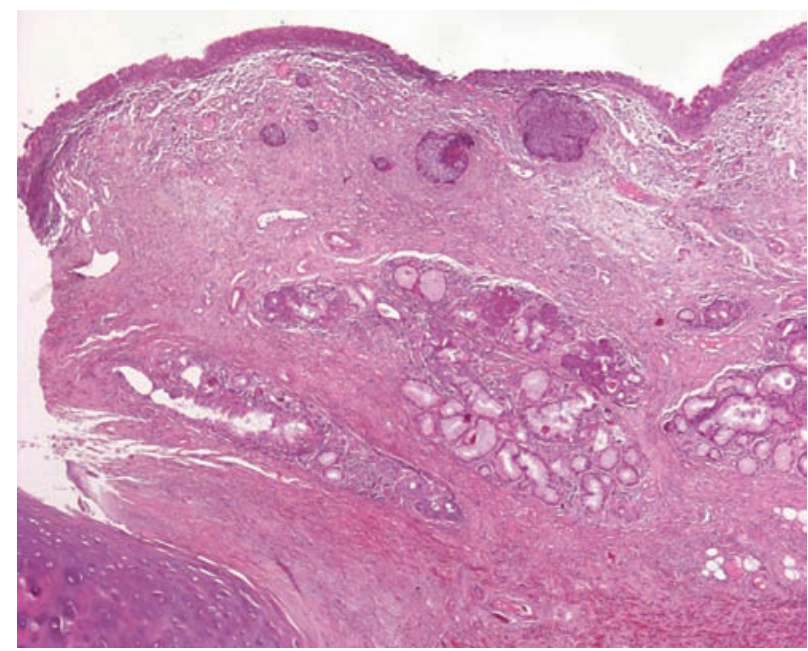

Figure 3 - Laryngotracheal mucosa.

\section{Discussion}

Squamous cell carcinomas of the UADT mostly develop as a single neoplastic event, although synchronous/metachronous lesions also occur ${ }^{2}$. Molecular studies have demonstrated that the primary tumor can be surrounded by a mucosal field made up of genetically altered cells ${ }^{3}$. Several theories have been postulated to explain this; the most accredited one in recent years identifies mucous alterations related to exposure to tobacco, alcohol, radiation, and HPV infection in genetically predisposed subjects ${ }^{4-7}$. This multistep process drives mucosal cells to uncontrolled proliferation and gain of genetic mutations, increasing the risk of independent and multifocal premalignant and malignant lesions. This pattern is known as field cancerization ${ }^{2,8,9}$.

Recent studies concerning the proteomic analysis of field cancerization in the pharynx and esophagus re- 
vealed changes in the protein profiles of normal mucosa similar to those in the tumors of the same patients ${ }^{10}$. This research may have a great impact on the improvement of personalized risk assessment in head and neck SCC.

The role of radiotherapy in causing field cancerization and a second SCC is controversial and remains to be clarified. According to Hashibe et al. ${ }^{11}$, patients treated with radiation only (RR $1.64,95 \% \mathrm{CI} 1.18-2.29$ ) or radiation with surgery (RR 1.49, 95\%CI 1.07-2.06) are at a higher risk of developing a second primary tumor than patients treated with surgery alone (RR 1.28, 95\%CI 0.93 -1.76). The risk becomes significant 10 years after irradiation. The same conclusion had been drawn earlier by Chen and $\mathrm{Hsu}^{12}$.

Conversely, Parker in 1988, Cooper in 1989 and Söderholm in 1994 analyzed consecutive series of patients affected by primary carcinomas of the UADT without reporting an increased risk in patients treated with radiotherapy instead of surgery ${ }^{6,13,14}$. Moreover, Rusthoven et al. ${ }^{15}$ in a retrospective case-control study including 27,985 patients even demonstrated that radiotherapy decreased the risk of a second tumor of the UADT, probably because it is able to destroy local foci of multifocal disease.

In the case reported here, the patient presented 3 important risk factors for field cancerization: alcohol use, cigarette smoke and previous radiotherapy. Moreover, the perfect match between the irradiation field and the extent of local tumor spread suggested the diagnosis of field cancerization. Instead, histopathology showed integrity of the entire mucosal surface but also widespread neoplastic embolization of the submucosal lymphatic vessels (Figures 1, 2 and 3). Field cancerization was therefore excluded, and the correct diagnosis was neoplastic lymphangiosis. This rare condition is sometimes seen in other malignancies such as papillary thyroid cancer or penile carcinoma in which the particular lymphophilia of the tumor cells provokes massive involvement of locoregional lymph nodes.

In conclusion, neoplastic lymphangiosis represents an adverse event and can simulate field cancerization. From a therapeutic point of view, the massive spread within the lymphatics makes it difficult to recognize adequate margins of resection. Therefore this condition must be looked upon as metastatic disease, and surgery as the primary approach has to be denied because of the impossibility to obtain reliable tumor-free margins. The prognosis in such locally advanced tumors is very poor; recognition of this clinical pattern may change the oncological approach from surgery to neoadjuvant treatment.

\section{References}

1. Mignogna MD, Fedele S, Lo Russo L, Mignogna C, Porter SR: Field cancerization in oral lichen planus. Eur J Surg Oncol, 33: 383-389, 2007.

2. Braakhius BJ, Tabor MP, Leemans CR, Van der Wall I, Snow GB, Brakenoff RH: Second primary tumors and field cancerization in oral and oropharyngeal cancer: molecular techiniques provide new insight and definitions. Head Neck, 24: 198-206, 2002.

3. Van Oijen MG, Slootweg PJ: Oral field cancerization: carcinogen induced independent events or micrometastatic deposits? Cancer Epidemiol Biomarkers Prev, 9: 249-256, 2000.

4. Strong MS, Incze J, Vaughan CW: Field cancerization in the aerodigestive tract-its etiology, manifestation and significance. J Otolaryngol, 3: 1-6, 1984.

5. Cooper JS, Pajak TF, Rubin P, Tupchong L, Brady LW, Leibel SA, Laramore GE, Marcial VA, Davis LW, Cox JD: Second malignancies in patients who have head and neck cancer: incidence, effect on survival and implications based on the RTOG experience. Int J Radiat Oncol Biol Phys, 17: 449-456, 1989.

6. Cloos J, Steen I, Timmerman AJ, Van der Schans GP, Snow G, Braakhius BJM: DNA-damage processing in blood lymphocytes of head and neck squamous cell carcinoma patients is dependent on tumor site. Int J Cancer, 68: 26-68, 1996.

7. Todd R, Donoff RB, Wong TW: The molecular biology of oral carcinogenesis: toward a tumor progression model. J Oral Maxillofacial Surg, 55: 613-623, 1997.

8. Braakhuis BJ, Tabor MP, Kummer JA, Leemans CR, Brakenhoff RH: A genetic explanation of Slaughter's concept of field cancerization: evidence and clinical implications. Cancer Res, 63: 1727-1730, 2003.

9. Papadimitrakopoulou VA, Shin DM, Hong WK: Molecular and cellular biomarkers for field cancerization and multistep process in head and neck tumorigenesis. Cancer Metastasis Rev,15: 53-76, 1996.

10. Roesch-Ely M, Leipold A, Nees M, Holzinger D, Dietz A, Flechtenmacher C, Wolf T, Zapatka M, Bosch F: Proteomic analysis of field cancerization in pharynx and oesophagus: a prospective pilot study. J Pathol, 221: 462-470, 2010.

11. Hashibe M, Ritz B, Le AD, Sankaranarayanan R, Zhang ZF: Radiotherapy for oral cancer as a risk factor for second primary cancers. Cancer Lett, 220: 185-195, 2005.

12. Chen CL, Hsu MM: Second primary epithelial malignancy of nasopharynx and nasal cavity afer successful curative radiation therapy of nasopharyngeal carcinoma. Hum Pathol, 31: 227-232, 2000.

13. Parker RG, Enstrom JE: Second primary cancers of the head and neck following treatment of initial primary head and neck cancers. Int J Radiat Oncol Biol Phys, 14: 561-564, 1988.

14. Söderholm AL, Pukkala E, Lindqvist C, Teppo L: Risk of new primary cancer in patients with oropharyngeal cancer. Br J Cancer, 9: 784-787, 1994.

15. Rusthoven K, Chen C, Raben D, Kavanagh B: Use of external beam radiotherapy is associated with reduced incidence of second primary head and neck cancer: a SEER database analysis. Int J Radiat Oncol Biol Phys, 71: 192-198, 2008. 